\title{
CORRECTION
}

\section{Correction to: Advances in PSMA-targeted therapy for prostate} cancer

Fujin Wang, Zhifeng Li, Xiaoqian Feng, Dazhuang Yang and Mei Lin (D)

(c) The Author(s), under exclusive licence to Springer Nature Limited 2021

Prostate Cancer and Prostatic Diseases (2022) 25:130; https://doi.org/10.1038/s41391-021-00421-5

Correction to: Prostate Cancer and Prostatic Diseases https://doi.org/10.1038/s41391-021-00394-5
In this article the affiliation details for Author Mei Lin were incorrectly given as ' 1,3 ' but should have been 3 .

The original article has been corrected. 